\title{
Special Issue: Advances in animation, rendering and visualization
}

The last decade has seen possibly the greatest advances in photorealistic rendering, the realistic modelling of complex objects, and the animation of very complex phenomena, as well as volumetric information processing, for example visualization. The advances are further supported by other developments in computer science, such as parallel architectures and the new algorithms that run on these machines.

Many of the recent scientific and technological advances are reflected in the seven technical papers and two survey papers collected in this special issue of Computer-Aided Design. Initial versions of these papers were presented at the 1st Computer Graphics Conference on Advanced Techniques in Animation, Rendering and Visualization held at Bilkent University, Turkey, in July 1993, in cooperation with the Technical and Scientific Research Council of Turkey. (TUBtTAK), the US Institute of Electrical and Electronics Engineers Turkey Section, and Bilkent University. The conference brought together close to 30 scientists from all over the world, most of them members of the leading research institutions in their fields.

Animation is a widely studied subject for many reasons. It is possibly the best visual technique for simulating the behaviour of almost anything that moves. It can generate entertaining scenes at high speeds, and thus the film and television industries are now almost dependent on various forms of computer animation. However, for the description of complex motions of complex objects, it is very difficult to obtain realtime motion on a nonspecialized computer. If photorealistic rendering is also requested, the problem is further complicated. The first two papers in this special issue discuss two different research areas in animation. 'Interactive body awareness', by Zhao and Badler, discusses the problem of self collision in the animation of human-like agents. The approach presented is also extended to handle collisions with environmental obstacles. 'Animation of deformable models', by Gïdïkbay and Ozgïç, discusses the problem of animating objects deformed under external forces. The techniques discussed are based on elasticity theory. Collision handling of objects, especially of membranes hitting solids, is examined.

Two popular rendering techniques, namely radiosity and ray tracing, are still widely researched, mostly because they generate very realistic images but at a high computational cost. One of the main trends of research in this area is the parallelization of these two algorithms. The third paper in the issue, 'Data management scheme for parallel radiosity', by Bountouch and Priol, discusses the usage of shared virtual memory in the parallel radiosity algorithm. It is advisable to read at the same time the first paper of the survey section, 'Parallel processing of the radiosity method', by Paddon and
Chalmers, to understand further the need for and importance of data and memory management in parallel radiosity algorithms. This critical survey/evaluation paper gives comparative descriptions of various parallel processing methods for the radiosity algorithm. The fourth paper, 'Efficient parallel spatial subdivision algorithm for object-based parallel ray tracing', by Aykanat, Işler and Orgüç, presents a subdivision algorithm that decomposes a given scene into rectangular regions adaptively so that the number of intersection tests (a major factor that increases the complexity of ray tracers) is reduced to a minimum. The following paper, 'RRIFF: a proposal for the interchange of realistic scene descriptions', by Guitton and Schlick, discusses the difficulties involved in the description of realistic rendering for the interchange of scenes. Then, a format is proposed which is independent of the rendering technique.

Scientific visualization is mostly associated with medical or other similar volume rendering research and applications. However, visualization techniques can indeed be applied to many other interesting phenomena, some even being conceptual in nature. The sixth paper, 'Chiron parallel program performance visualization system', by Goosen, Karlin, Cheriton and Polkin, demonstrates the use of scientific visualization techniques to assist programmers working on parallel systems to visualize their performance, especially in shared memory environments. The application of visualization techniques coupled with numerical methods to the generation of visual numerical environments is proposed in the paper 'Visual numerical environment: reference models, methods and tools', by Spitaleri. The second survey paper, 'Survey of parallel approaches to scientific visualization', by Whitman, discusses and presents developments in the parallel visualization of scientific simulation data.

This special issue brings together nine interesting papers on topics ranging from computer graphics to visualization techniques applied to abstract data, coupled with advanced software tools. Most of the techniques discussed are powerful tools for the CAD researcher.

I would like to thank all the authors of the papers in this issue for carefully revising their conference presentations. I would like to express my special thanks to my colleague Dr Varol Akman for his most valuable efforts in chairing the programme committee and the refereeing process. Many thanks are due to Professor Les Piegl, who has provided valuable support in the revision process, and to Ms Karen Hemingway, who has done all the organizational work in getting this issue published.

\author{
Bülent Özgüç \\ Bilkent University \\ Turkey
}

